\title{
Paediatric outcomes and timing of admission
}

\author{
Louise Ramsden, ${ }^{1}$ Martin Patrick McColgan, ${ }^{2}$ Thomas Rossor, ${ }_{1}^{3,4}$ Anne Greenough, 2,3,4,5 \\ Simon J Clark ${ }^{1,2}$
}

- Additional material is published online only. To view please visit the journal online (http://dx.doi.org/10.1136/ archdischild-2017-314559).

1 Neonatal Unit, Sheffield Teaching Foundation Hospitals Trust, Sheffield, UK

${ }^{2}$ Royal College of Paediatrics and Child Health, London, UK ${ }^{3}$ MRC-Asthma UK Centre in Allergic Mechanisms of Asthma, King's College London, London, UK

${ }^{4}$ Department of Women and Children's Health, School of Life Course Sciences, Faculty of Life Sciences and Medicine, King's College London, London, UK ${ }^{5}$ NIHR Biomedical Centre at Guy's and St Thomas NHS Foundation Trust and King's College London, London, UK

\section{Correspondence to Dr Simon J Clark: Simon.Clark@sth.nhs.uk}

Received 12 December 2017 Revised 12 February 2018 Accepted 14 February 2018 Published Online First 15 March 2018
Check for updates

To cite: Ramsden $\mathrm{L}$, McColgan MP, Rossor T, et al. Arch Dis Child

2018:103:611-617.

\section{ABSTRACT}

Studies of adult patients have demonstrated that weekend admissions compared with weekday admissions had a significantly higher hospital mortality rate. We have reviewed the literature to determine if the timing of admission, for example, weekend or weekday, influenced mortality and morbidity in children. Seventeen studies reported the effect of timing of admission on mortality, and only four studies demonstrated an increase in those admitted at the weekend. Meta-analysis of the results of 15 of the studies demonstrated there was no significant weekend effect. There was, however, considerable heterogeneity in the studies. There were two large UK studies: one reported an increased mortality only for planned weekend admissions likely explained by planned admissions for complex conditions and the other showed no significant weekend effect. Two studies, one of which was large $(n=2913)$, reported more surgical complications in infants undergoing weekend oesophageal atresia and trachea-oesophageal repair. Medication errors have also been reported to be more common at weekends. Five studies reported the effect of length of stay, meta-analysis demonstrated a significantly increased length of stay following a weekend admission, the mean difference was approximately 1 day. Those data, however, should be interpreted with the caveat that there was no adjustment in all of the studies for differences in disease severity. We conclude that weekend admission overall does not increase mortality but may be associated with a longer length of stay and, in certain conditions, with greater morbidity.

\section{INTRODUCTION}

In 2001, it was reported that weekend admissions when compared with weekday admissions of 3789917 adults and children admitted through an emergency department to an acute care hospital in Canada had a significantly higher inhospital mortality rate if they had a ruptured abdominal aortic aneurysm, acute epiglottis or pulmonary embolism. ${ }^{1}$ The differences persisted for all diagnoses after adjustment for age, sex and coexisting disorders. ${ }^{1}$ A subsequent retrospective analysis of deaths of all 14217640 admissions to the English National Health Service during the financial year 2009/2010 demonstrated that 30-day mortality was increased in those admitted on weekend, although the likelihood of death occurring was less on a weekend than on a midweek day. ${ }^{2}$ More recently, an analysis of deaths of 503938 unselected emergency admissions to four UK hospitals was undertaken. Deaths within 30 days of admission occurred in $18313(4.7 \%)$ patients admitted on a weekday and $6070(5.1 \%)$ patients admitted on a weekend
$(\mathrm{P}<0.001)$. Adjustment for routine haematology and biochemistry tests substantially reduced the excess mortality associated with emergency admissions at weekends and public holidays. No hospital workload measure was independently associated with mortality. Adjustment for patient level factors was not available in the study, but it was postulated that such an adjustment might have further reduced the residual excess mortality, as this was clustered around midday at weekends. ${ }^{3}$

The infant mortality rate in England and Wales is 3.6 per 1000 live births and the child mortality rate is 1 per $1000,{ }^{4}$ both are much lower than the adult mortality rate. Nevertheless, it is important to assess whether there is a weekend effect on mortality and other adverse outcomes in paediatric populations. We, therefore, have reviewed the literature to identify if there were significant differences according to admission at the weekend or weekdays regarding mortality, procedure complications, length and cost of hospital stay, medication errors and unplanned readmission rates. These are important questions to address as the results may have implications for redirection of health services resources to reduce adverse outcomes.

\section{Mortality}

Seventeen studies have reported the effect of timing of admission on mortality. ${ }^{5-21}$ Only four of the studies 591217 demonstrated an increased mortality in those at the weekend. One study ${ }^{16}$ showed among 234192 admissions to paediatric intensive care unit (PICU) in the USA, despite off hours admissions having higher acuity, after adjustment such admissions had a significantly lower odds of mortality (OR 0.91, 95\% CI 0.86 to 0.95$)$. Similarly, examination of 4456 emergency admissions to a PICU in an Australian hospital demonstrated that the risk-adjusted mortality was lower in after-hours (OR 0.712, $95 \%$ CI 0.518 to 0.980 ). Inhours were 08:00 18:00 on Monday-Friday and 08:00 to $12: 00$ on Saturday and Sunday, while after-hours were all other times. ${ }^{18}$ Meta-analysis was undertaken of the results of 15 of the studies (see online supplement for information regarding the analysis). This demonstrated a significant increased risk of mortality at the weekend (typical relative risk (RR) 1.37 (95\% CI 1.07 to 1.76 )) (figure 1), but the effect was no longer significant once adjusted data were analysed (figure 2). Importantly, however, different adjustments were used in the various studies, and there was considerable heterogeneity between the studies (table 1). This included the comparator time periods during the week, for 


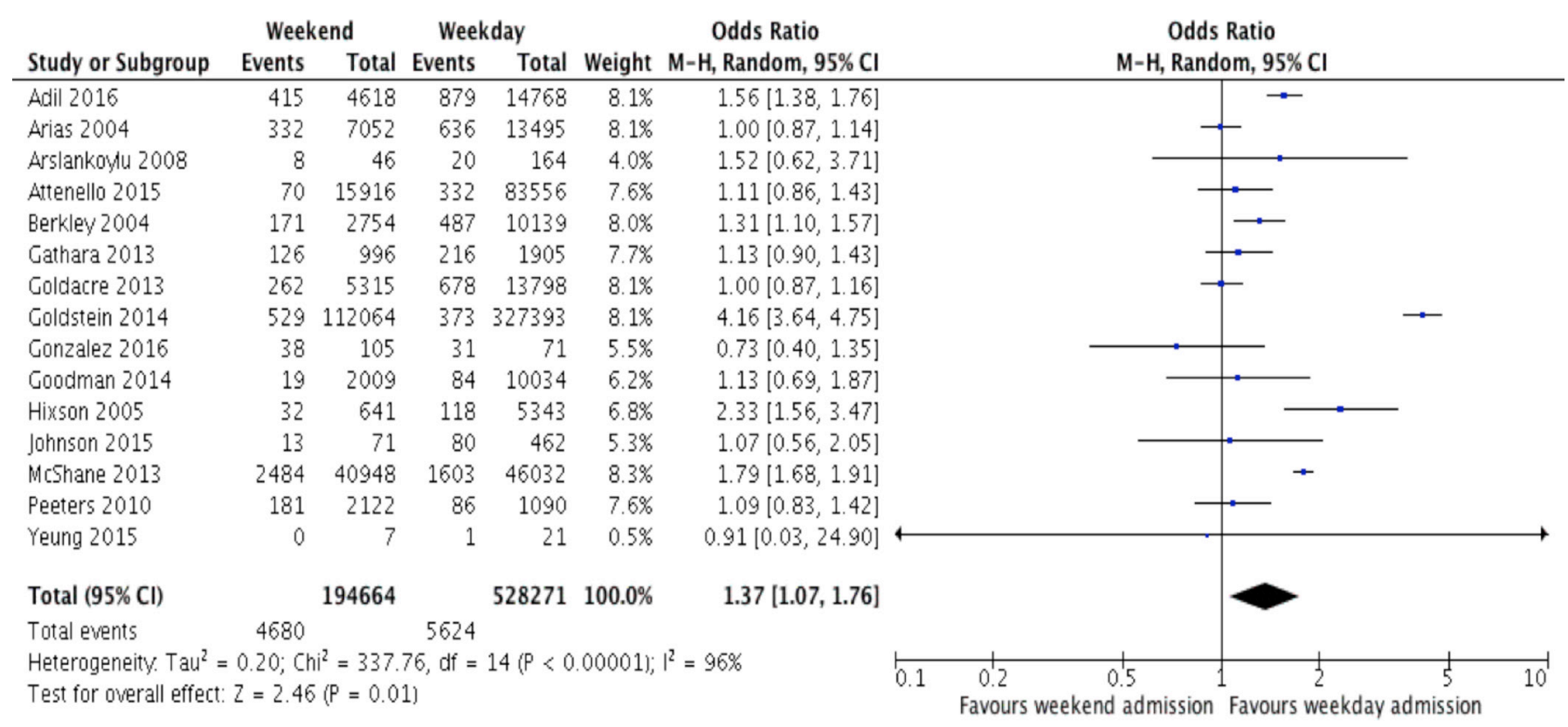

Figure 1 Weekend effect on mortality during admission (unadjusted).

example, the length of the weekend varied from 19:00 on Friday to 07:00 on Monday compared with Saturday 12:00 to Sunday 24:00. Others compared out of hours with regular hours, which were 07:00-19:00 on weekdays; another definition of out of hours was nights, weekends and public holidays. This may have influenced the results as, for example, in one study of 20547 PICU admissions, although there was no weekend effect on mortality, there was an excess of mortality in children admitted during the evening compared with during daytime hours. ${ }^{6}$ This was not confirmed in another study, but it was much smaller $(n=210)^{7}$

Of those that showed a significant adverse weekend effect, one assessed 19386 children aged 1-18 years of age in the USA who were admitted with ischaemic or haemorrhagic stroke. ${ }^{5}$ A limitation of the study was that it relied on the correct coding of the diagnoses in the database. ${ }^{5}$ Another examined the outcome of 12893 children older than or equal to 90 days admitted to a rural hospital in Kenya. Although deaths within 4 hours and 4-48 hours of admission were significantly higher at the weekend, deaths beyond 48 hours were not. ${ }^{9}$ A further study examined 439457 patients less than 18 years of age who required surgery on the same day of admission. After adjusting for confounding variables at the weekend, the OR for the risk of death was 1.63 (95\% CI 1.21 to 2.2$).{ }^{12}$ Two large studies reported UK findings. In one, ${ }^{17}$ a nationwide paediatric intensive care audit was undertaken of 86000 admissions. Nearly half $(47.1 \%)$ of the admissions were out of hours and $79.2 \%$ of those were emergency admissions. The risk adjusted mortality for planned admissions was OR 1.99 (1.67-2.37), but there was no significant risk for emergency admissions (OR 0.93, $95 \%$ CI 0.86 to 1.01 ). The overall raised mortality for out of hours admissions was suggested by the authors to be accounted for by planned admissions following complex operations. ${ }^{17}$ In the other large UK-based study, 19729 patients were included with 12697 being under 15 years of age. The case-related fatality rates following admission for meningococcal disease were reported. ${ }^{11}$ No significant differences were reported between weekend and weekday admissions and, after undertaking sensitivity analyses and analysing multivariate models, the lack of a weekend effect remained.

Odds Ratio

Odds Ratio

Study or Subgroup log[Odds Ratio] SE Weight IV, Random, $95 \% \mathrm{Cl}$ IV, Random, $95 \% \mathrm{Cl}$

\begin{tabular}{|c|c|c|c|c|}
\hline Adil 2016 & 0.1823 & 0.275 & $3.5 \%$ & $1.20[0.70,2.06]$ \\
\hline Adil 2016 & 0.3365 & 0.123 & $10.4 \%$ & $1.40[1.10,1.78]$ \\
\hline Attenello 2015 & 0.0953 & 0.1315 & $9.8 \%$ & $1.10[0.85,1.4$ \\
\hline Gathara 2013 & 0.1222 & 0.1161 & $11.0 \%$ & $1.13[0.90,1.4$ \\
\hline Goldacre 2013 & -0.0202 & 0.1165 & $11.0 \%$ & $0.98[0.78,1.2$ \\
\hline Goldstein 2014 & 0.4886 & 0.152 & $8.3 \%$ & $1.63[1.21,2.2$ \\
\hline Goodman 2014 & 0 & 0.1139 & $11.2 \%$ & $1.00[0.80,1.2$ \\
\hline McShane 2013 & 0.0488 & 0.0378 & $19.0 \%$ & $1.05[0.98,1.1$ \\
\hline Numa 2008 & -0.3397 & 0.1623 & $7.6 \%$ & $0.71[0.52,0.9$ \\
\hline Peeters 2010 & 0.0296 & 0.1551 & $8.1 \%$ & $1.03[0.76,1.4$ \\
\hline $5 \% \mathrm{Cl})$ & & & $100.0 \%$ & $1.09[0.98,1.2$ \\
\hline \multicolumn{5}{|c|}{$\begin{array}{l}\text { Heterogeneity. Tau }{ }^{2}=0.02 ; \mathrm{Chi}^{2}=20.41, \mathrm{df}=9\left(\mathrm{P}=0.02 \mathrm{l} ; \mathrm{I}^{2}=56 \%\right. \\
\text { Test for overall effect: } \mathrm{Z}=1.57(\mathrm{P}=0.12)\end{array}$} \\
\hline
\end{tabular}

Figure 2 Weekend effect on adjusted mortality during admission. 


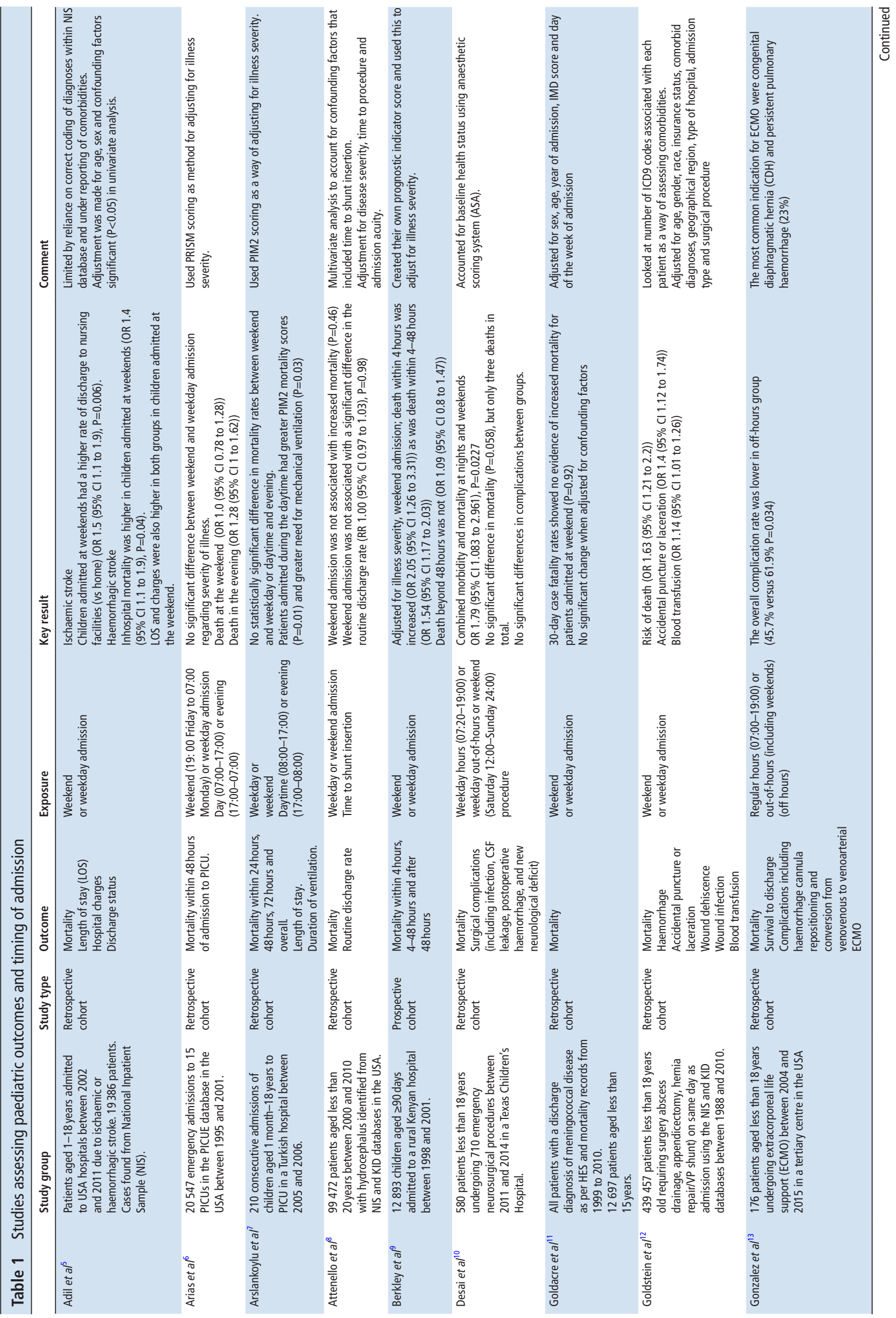




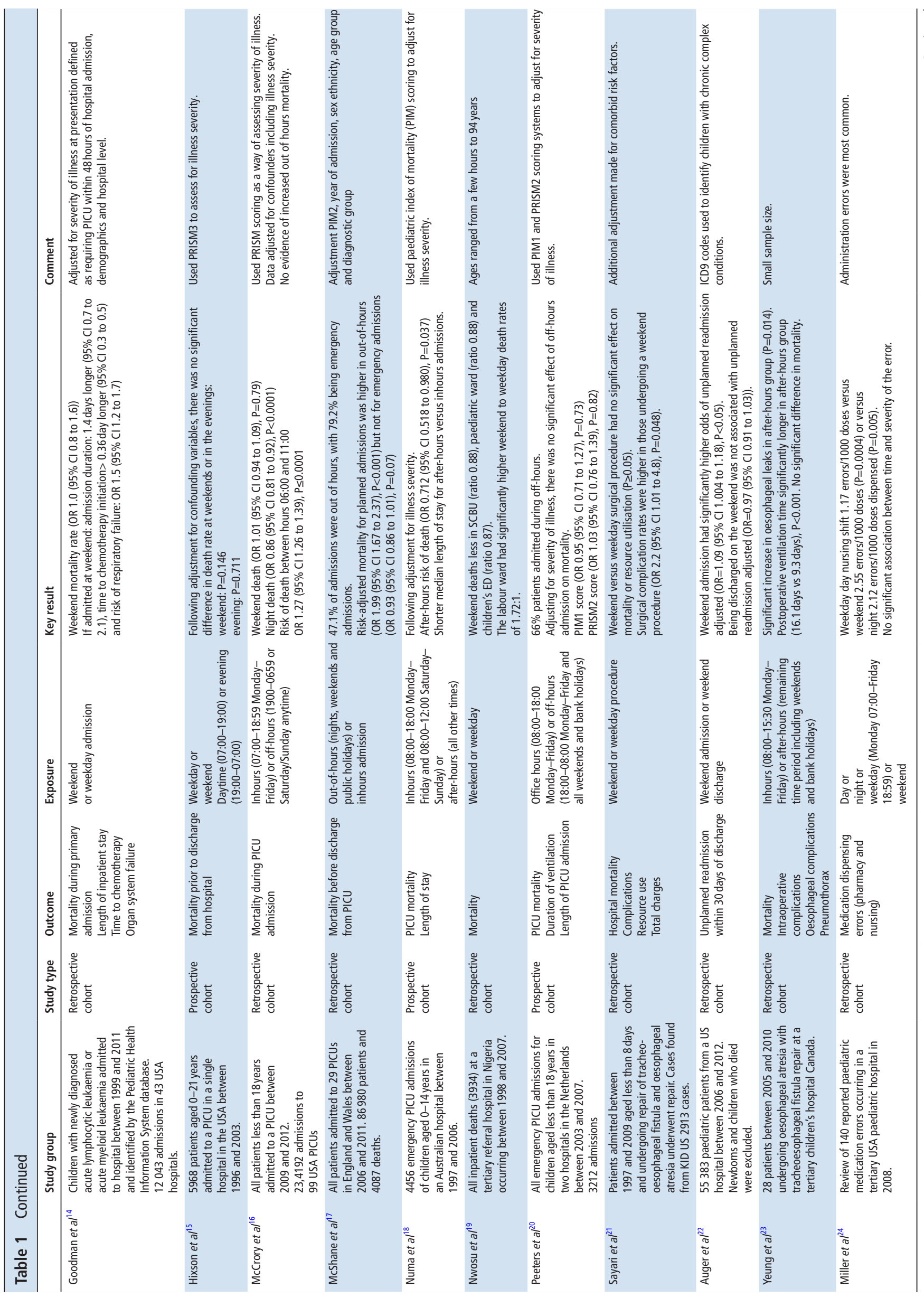

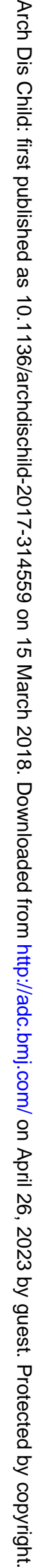




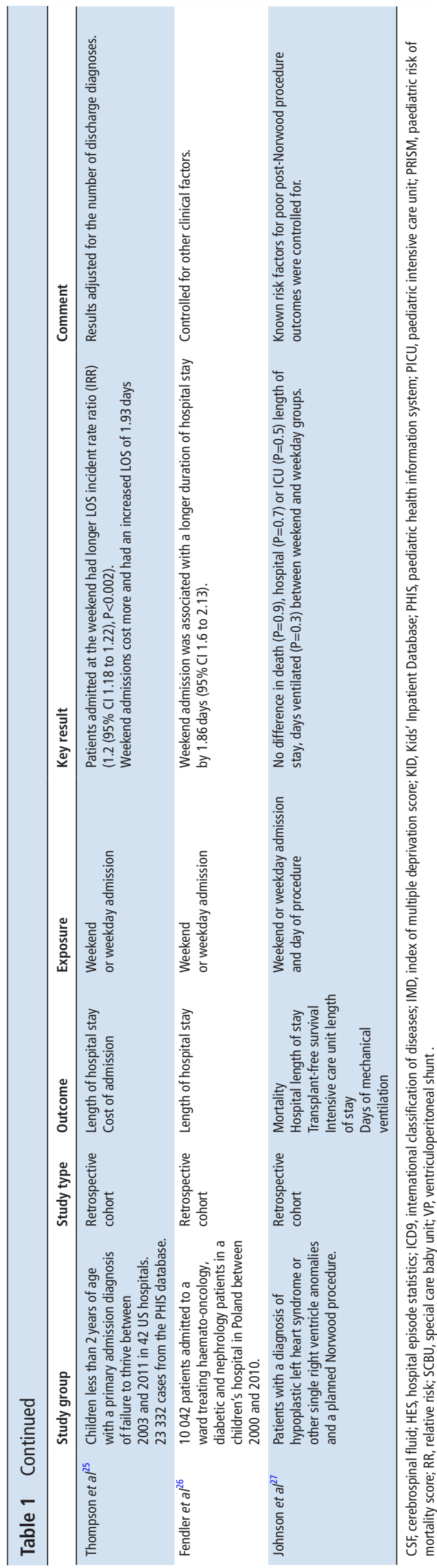

Complication rates

A number of studies have assessed whether complications were increased at the weekend. The complications included increased unplanned readmission rates, ${ }^{22}$ increased complication rates, ${ }^{1012} 132123$ treatment delay ${ }^{14}$ and increased medication errors. ${ }^{24}$ In a study of 55383 children in the USA (newborns and children who died were excluded), weekend admission was associated with a significantly higher odds of an unplanned readmission in 30 days, but weekend discharge was not associated with a significantly higher unplanned readmission rate. ${ }^{22}$ There appears to be a weekend effect regarding certain procedural complications. In a retrospective cohort of 580 patients less than 18 years of age undergoing 710 urgent or emergency neurosurgical procedures in a Texas Children's Hospital, there was a significant excess of surgical complications at weekends and weekdays after-hours. ${ }^{10}$ In a small study of 28 patients undergoing oesophageal atresia and trachea-oesophageal repair, there were significantly more oesophageal leaks in those admitted after-hours ${ }^{23}$ and this was associated with a longer postoperative length of ventilation (16.1 vs 9.3 days). ${ }^{23}$ Those findings ${ }^{23}$ were confirmed in a larger cohort $(n=861)$ of infants less than 8 days of age undergoing oesophageal atresia and trachea-oesophageal repair identified from the USA Kid's Inpatient Database. A greater rate of surgical complications were reported in those undergoing a weekend procedure (OR 2.2, 95\% CI 1.01 to 4.8 ).$^{21}$ In a study of 439457 children less than 18 years of age in the USA who required surgery on the day of admission, there was an increase in procedural complications and receipt of blood transfusions despite similar intraoperative haemorrhage at the weekend. ${ }^{12}$ In contrast, the overall complication rate was lower out of hours in 176 children requiring extracorporeal membrane oxygenation in a tertiary centre. ${ }^{13}$ Weekend admission was not associated with a significant excess of mortality among 12043 children with newly diagnosed acute lymphocytic leukaemia or acute myeloid leukaemia, but the length of admission was on average 1.4 days longer and the time to start chemotherapy was 0.3 days later (both differences were statistically significantly). ${ }^{14}$ In addition, children had an increased risk of respiratory failure if admitted at the weekend (OR 1.5, 95\% CI 1.2 to 1.7$).{ }^{14}$ In a report of 140 medication errors occurring in a tertiary paediatric hospital, the rate of errors per 1000 doses were significantly higher on weekend compared with week days $(2.25$ vs $1.17, \mathrm{P}=0.004) .^{24}$

\section{Length of stay}

Five studies have reported the effect of timing of admission on length of stay ${ }^{14}$ 18-27 $^{25}$ A number of studies reported an increased length of stay in those admitted at the weekend, but it is important to appreciate that this was not the case in all studies, ${ }^{1827}$ and the populations assessed in the various studies were very heterogenous. In 23332 children aged less than 2 years admitted with a diagnosis of failure to thrive to USA hospitals, weekend admission was significantly correlated with increased length of stay. The average increase in the length of stay of 1.93 days and was associated with an increase in the cost of care. ${ }^{25}$ Among 10042 patients admitted to a ward in a children's hospital in Poland that treated heamato-oncology, diabetic and nephrology patients, after controlling for clinical factors, weekend admission was associated with a longer duration of hospital stay by on average 1.86 days. $^{26}$ In contrast, in 549 patients undergoing a planned Norwood procedure with a diagnosis of hypoplastic left heart syndrome or other single right ventricle anomalies in a children's hospital in Chicago, after adjusting for known risk factors for poor outcomes, there was no significant effect on length of 


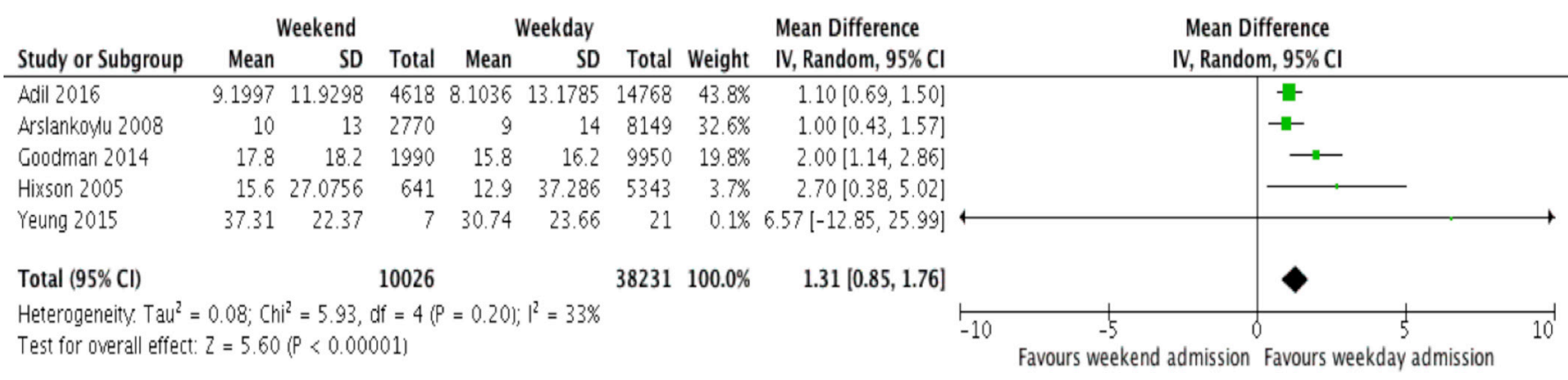

Figure 3 Weekend effect on length of hospital stay.

stay, length of PICU stay, duration of ventilation or mortality. ${ }^{27}$ Furthermore, another study of PICU patients demonstrated that among 4456 emergency PICU admissions of children less than 14 years of age, after using paediatric index of mortality (PIM) scoring to adjust for illness severity, the median length of stay was shorter after out of hours admission. ${ }^{18}$ Meta-analysis demonstrated an increased length of stay following weekend admission (RR 1.31, 95\% CI 0.85 to 1.76 ); the mean difference was approximately 1 day (figure 3 ). The results, however, should be interpreted with caution as adjustment could not be made in all studies for differing illness severity in patients admitted out of hours or at weekends.

\section{DISCUSSION}

We have not found a significant 'weekend' effect on paediatric mortality but an adverse effect on length of stay and greater complications in certain conditions. Our literature review emphasises the heterogeneity of the studies examining this topic. It was, however, possible to undertake a meta-analysis of the majority of studies on the impact on mortality of weekend admission that demonstrated no significant effect on mortality when adjusted results were used. It is important, however, to re-emphasise that different adjustments were used in the various studies.

The majority of studies have assessed the outcomes of children living and being treated outside the UK and, therefore, the results must be interpreted with the caveat that they may have been in the context of different healthcare provision. In one of the two large UK studies, ${ }^{11}$ no significant differences were reported between weekend and weekday admissions and in the other ${ }^{17}$ an increase in mortality was seen only in planned weekend admissions and not emergency weekend admissions. Those results ${ }^{17}$ highlight that it may be case mix that influences results with patients following complex surgery being more likely to be admitted to PICU at the weekend.

We have not reported whether deaths were more likely to occur at the weekend or on a weekday, as this was not available for the majority of studies. Importantly, the retrospective analysis of deaths of all 14,217,640 admissions to the English National Health Service demonstrated that the 30 day mortality was increased in those admitted on weekend days, but the likelihood of death occurring was less on a weekend day than on a mid-week day. ${ }^{2}$ It would be important to determine in UK paediatric populations the timing of deaths to identify if there are any excess deaths at the weekend as that might reflect inadequate 'resource' at that time.

None of the studies specifically looked at staffing levels, which could contribute to a 'weekend effect'. Paediatric staffing is an area of concern, $89 \%$ of consultants responding to a Royal College of Paediatrics and Child Health (RCPCH) survey stated that they were very or moderately concerned about how the service would cope in the next 6 months. ${ }^{28}$ The Royal College of Nursing ${ }^{29}$ and the $\mathrm{RCPCH}^{30}$ have issued documents detailing standards of staffing and expectations of care for children admitted to hospital. An audit of the medical standards by $\mathrm{RCPCH},{ }^{31} 2$ years after their introduction, found that, although they were being met in the majority of cases, there were still improvements to be made to better achieve the standards out-ofhours. The weekend effect may be mitigated by paediatrics being a relatively senior-led specialty; $96.4 \%$ of units surveyed in 'Consultant Delivered Care' operated some form of consultant delivered care, including $90.3 \%$ of consultant-led handovers. ${ }^{32}$

In conclusion, studies of adult patients had demonstrated a significantly higher in hospital mortality in those admitted at weekends. A recent study, however, suggested that adjustment for acuity reduced that risk. Our review of paediatric studies demonstrated no overall significant adjusted risk for mortality following weekend admissions. There was, however, an increase in complications following weekend procedures in certain conditions. In five studies, there was an increased length of stay following a weekend admission, but it was not possible to adjust in all for patient acuity. The increase in hospital stay and apparent excess in complications in certain conditions in infants and children admitted at the weekend needs further investigation. Those results would identify whether there is a need for redirection of health services resources to reduce adverse outcomes in infants and children.

Contributors LR, MPM and SJC undertook the literature search, and TR and AG undertook the analysis. All authors were involved in the preparation of the manuscript and approved the final version.

Funding This research was supported by the National Institute for Health Research (NIHR) Biomedical Research Centre at Guy's and St Thomas' NHS Foundation Trust and King's College London.

Disclaimer The views expressed are those of the authors and not necessarily those of the NHS, the NIHR or the Department of Health.

Competing interests None declared.

Provenance and peer review Not commissioned; externally peer reviewed. Data sharing statement We agree to sharing data if requested.

(c) Article author(s) (or their employer(s) unless otherwise stated in the text of the article) 2018. All rights reserved. No commercial use is permitted unless otherwise expressly granted.

\section{REFERENCES}

1 Bell CM, Redelmeier DA. Mortality among patients admitted to hospitals on weekends as compared with weekdays. N Engl J Med 2001;345:663-8.

2 Freemantle N, Richardson M, Wood J, et al. Weekend hospitalization and additional risk of death: an analysis of inpatient data. J $R$ Soc Med 2012;105:74-84.

3 Walker AS, Mason A, Quan TP, et al. Mortality risks associated with emergency admissions during weekends and public holidays: an analysis of electronic health records. Lancet 2017;390:62-72. 
4 Office for National Statistics 2016. Childhood mortality rates in England and Wales, 2014. Published online April 2016.

5 Adil MM, Vidal G, Beslow LA. Weekend effect in children with stroke in the nationwide inpatient sample. Stroke 2016;47:1436-43.

6 Arias Y, Taylor DS, Marcin JP. Association between evening admissions and higher mortality rates in the pediatric intensive care unit. Pediatrics 2004;113:e530-4.

7 Arslankoylu AE, Bayrakci B, Oymak Y, et al. Admission time and mortality rates. Indian J Pediatr 2008;75:691-4.

8 Attenello FJ, Christian E, Wen T, et al. Reevaluating the weekend effect on patients with hydrocephalus undergoing operative shunt intervention. J Neurosurg 2016:17:156-62.

9 Berkley JA, Brent A, Mwangi I, et al. Mortality among Kenyan children admitted to a rural district hospital on weekends as compared with weekdays. Pediatrics 2004;114:1737-8.

10 Desai V, Gonda D, Ryan SL, et al. The effect of weekend and after-hours surgery on morbidity and mortality rates in pediatric neurosurgery patients. J Neurosurg Pediatr 2015:16:726-31.

11 Goldacre MJ, Maisonneuve JJ. Mortality from meningococcal disease by day of the week: English national linked database study. J Public Health 2013:35:413-21.

12 Goldstein SD, Papandria DJ, Aboagye J, et al. The "weekend effect" in pediatric surgery - increased mortality for children undergoing urgent surgery during the weekend. J Pediatr Surg 2014;49:1087-91.

13 Gonzalez KW, Dalton BG, Weaver KL, et al. Effect of timing of cannulation on outcome for pediatric extracorporeal life support. Pediatr Surg Int 2016:32:665-9.

14 Goodman EK, Reilly AF, Fisher BT, et al. Association of weekend admission with hospital length of stay, time to chemotherapy, and risk for respiratory failure in pediatric patients with newly diagnosed leukemia at freestanding US children's hospitals. JAMA Pediatr 2014:168:925-31.

15 Hixson ED, Davis S, Morris S, et al. Do weekends or evenings matter in a pediatric intensive care unit? Pediatr Crit Care Med 2005;6:523-30.

16 McCrory MC, Gower EW, Simpson SL, et al. Off-hours admission to pediatric intensive care and mortality. Pediatrics 2014;134:e1345-53.

17 McShane P, Draper ES, McKinney PA, et al. Effects of out-of-hours and winter admissions and number of patients per unit on mortality in pediatric intensive care. J Pediatr 2013:163:1039-44.

18 Numa A, Williams G, Awad J, et al. After-hours admissions are not associated with increased risk-adjusted mortality in pediatric intensive care. Intensive Care Med 2008;34:148-51.
19 Nwosu BO, Eke NO, Obi-Nwosu A, et al. Weekend versus weekday hospital deaths: analysis of in-patient data in a Nigerian tertiary healthcare center. Niger I Clin Pract 2013;16:501-4.

20 Peeters B, Jansen NJ, Bollen CW, et al. Off-hours admission and mortality in two pediatric intensive care units without 24-h in-house senior staff attendance. Intensive Care Med 2010;36:1923-7.

21 Sayari AJ, Tashiro J, Wang B, et al. Weekday vs. weekend repair of esophageal atresia and tracheoesophageal fistula. J Pediatr Surg 2016:51:739-42.

22 Auger KA, Davis MM. Pediatric weekend admission and increased unplanned readmission rates. J Hosp Med 2015;10:743-5.

23 Yeung A, Butterworth SA. A comparison of surgical outcomes between in-hours and after-hours tracheoesophageal fistula repairs. J Pediatr Surg 2015;50:805-8.

24 Miller AD, Piro CC, Rudisill CN, et al. Nighttime and weekend medication error rates in an inpatient pediatric population. Ann Pharmacother 2010;44:1739-46.

25 Thompson RT, Bennett WE, Finnell SM, et al. Increased length of stay and costs associated with weekend admissions for failure to thrive. Pediatrics 2013;131:e80 $5-\mathrm{e} 810$.

26 Fendler W, Baranowska-Jazwiecka A, Hogendorf A, et al. Weekend matters: Friday and Saturday admissions are associated with prolonged hospitalization of children. Clin Pediatr 2013;52:875-8.

27 Johnson JT, Sleeper LA, Chen S, et al. Associations between day of admission and day of surgery on outcome and resource utilization in infants with hypoplastic left heart syndrome who underwent stage I Palliation (from the Single Ventricle Reconstruction Trial). Am J Cardiol 2015;116:1263-9.

28 Royal College of Paediatrics and Child Health. Paediatric rota gaps and vacancies 2016. 5-11 Theobalds Road, London: Royal College of Paediatrics and Child Health, 2015.

29 Royal College of Nursing. Defining staffing levels for children and young people's services. Cavendish Square, London: Royal College of Nursing, 2013.

30 Royal College of Paediatrics and Child Health. Facing the future: standards for paediatric services. 5-11 Theobalds Road, London: Royal College of Paediatrics and Child Health, 2011.

31 Royal College of Paediatrics and Child Health. Back to facing the future: an audit of acute paediatrics service standards in the UK. Executive summary. 5-11 Theobalds Road, London: Royal College of Paediatrics and Child Health, 2013.

32 Royal College of Paediatrics and Child Health. Consultant delivered care. An evaluation of new ways of working in Paediatrics. 5-11 Theobalds Road, London: Royal College of Paediatrics and Child Health, 2012. 